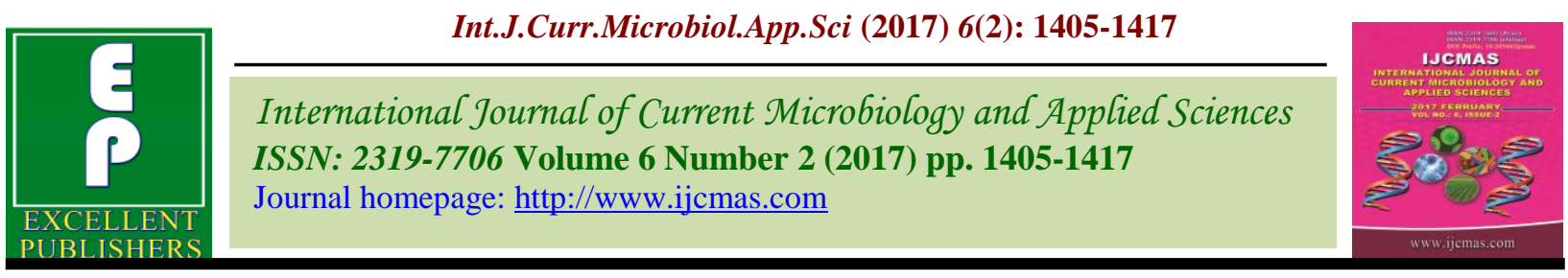

Original Research Article http://dx.doi.org/10.20546/ijcmas.2017.602.159

\title{
Bioefficacy and Safety of Cyantraniliprole $10 \%$ (W/V) OD Against Sucking Pests in Cotton
}

\author{
P. Karthik ${ }^{1}$, T.Thiruveni ${ }^{1}$, K.Indirakumar ${ }^{1}$, K. Gunasekaran ${ }^{1}$, \\ S. Kuttalam ${ }^{1}$ and V.M.Srinivasan ${ }^{2}$ \\ ${ }^{1}$ Department of Agricultural Entomology, TNAU, Coimbatore, India \\ ${ }^{2}$ Department of Plant Pathology, TNAU, Coimbatore, India \\ *Corresponding author
}

\begin{tabular}{|c|c|}
\hline & A B S T R A C T \\
\hline $\begin{array}{l}\text { Ke y w o r d s } \\
\text { Bioefficacy, Safety, } \\
\text { New insecticide, } \\
\text { Sucking insect pest, } \\
\text { Natural enemy. }\end{array}$ & \multirow{3}{*}{$\begin{array}{l}\text { Cyantraniliprole } 10 \% \text { OD at } 90 \mathrm{~g} \text { a.i.ha }{ }^{-1} \text { was significantly effective when } \\
\text { sprayed twice at } 15 \text { days interval, minimized the sucking pests population } \\
\text { and increased cotton kapas yield. Cyantraniliprole } 10 \% \text { OD treatments at } 90 \text {, } \\
180 \text { and } 320 \mathrm{~g} \text { a.i. ha }{ }^{-1} \text { concentrations had not caused any phytotoxic effects } \\
\text { like injury to leaf tip, leaf surface, wilting, vein clearing, necrosis, epinasty } \\
\text { and hyponasty. Cyantraniliprole } 10 \% \text { (w/v) OD showed the least effect } \\
\text { against the spiders. Spider's population in cotton ecosystem showed } \\
\text { considerable decrease initially in all the treatments, it started increasing in } \\
\text { later. }\end{array}$} \\
\hline Article Info & \\
\hline $\begin{array}{l}\text { Accepted: } \\
\text { 24 January } 2017 \\
\text { Available Online: } \\
\text { 10 February } 2017\end{array}$ & \\
\hline
\end{tabular}

\section{Introduction}

Cotton (Gossypium spp.), is an important agricultural commodity, traded all over the world. In general, the conditions required for the cultivation of cotton are met within the seasonally dry tropics and subtropics in the Northern and Southern hemispheres. India has progressed substantially in improving both production and productivity of cotton over the last five years, transforming from a net importer of cotton, to becoming one of the largest exporters, shipping 5.5 million bales in 2010-11, second only to the USA. About 70 per cent of the global cotton production comes from four countries, which include China (27\%), India (22\%), USA (13\%) and Pakistan $(8 \%)$. Cotton cultivation is a very important part of the Indian agrarian landscape and provides sustainable livelihood to a sizeable population in India. It is cultivated about 10.31 million hectares in the country, which accounts for 30 per cent of the global cotton area and contributes to 22 per cent of the global cotton production. It is estimated that more than 5.8 million farmers cultivate cotton in India and about 40-50 million people are employed directly or indirectly by the cotton industry. Cotton is cultivated about 1.21 lakh hectares in Tamil Nadu (Anonymous, 2012). The yield loss to cotton due to insect pests is 87 per cent (Taley et al., 1988). Nearly 1,326 insects and mites all over the world and about 200 insects in 
India have been documented of which ten are economically important in Tamil Nadu (Venugopal, 1998). Major sucking pests consisting of Amrasca biguttula biguttula, Aphis gossypii, Thrips tabaci and Bemisia tabaci inflict heavy damage.

Chemical insecticides are used as the frontline defence sources against these insect pests, in spite of their drawbacks and the cotton growers in India depend heavily on synthetic pesticides to combat pests and it has been estimated that cotton consumes about 54 per cent of the total insecticides used in the country (Anonymous, 1997). Indiscriminate use of organophosphates, carbamates and synthetic pyrethroids has created a number of problems such as pests developing resistance to insecticides (McCaffery et al., 1989; Armes et al., 1994), pest resurgence (Hardin et al., 1995) and bioconcentrations of pesticide residues in consumable produce at harvest (Rolando et al., 1982). Among the harmful effects of insecticides, persistence of toxic pesticide residues in plants, soil and water are of great concern for consumer's health and safety to animals. It is estimated that more than 50 per cent of applied insecticides reach the soil and water bodies during application (Awasthi et al., 2002).

As chemical pesticides continue to be the mainstay of most of the pest control programmes. At the same time, to overcome the above problems, identification of new molecules are needed to combat the associated drawbacks. At present, the golden age of insecticide research has come with selective, neuro active and easily degradable compounds. These newer molecules always have a higher stability and superiority over conventional pesticides to control the pest population in a classical manner at field level. In this array, newer groups with higher insecticidal property, selective action and lower mammalian toxicity fits well in the integrated pest management (IPM) concept In IPM programmes, insecticides having selectivity, favouring non-target organisms like parasitoids and predators are highly preferred. Formulations that are safe to the applicant and more environment friendly are encouraged. One such new molecule, cyantraniliprole $10 \%$ OD is a novel class of insecticide having a unique chemical structure used against broad spectrum of insects and safe to non-target insects (parasitoids, predators and pollinators) and acts on ryanodine receptor modulator. It is also used to control insects which are found resistant to other insecticides and fits into IPM programs. With the above background, investigations were carried out on cyantraniliprole $10 \%$ OD, a new formulation.

\section{Materials and Methods}

\section{In-vivo evaluation}

Cyantraniliprole 10\% OD was tested along with eight treatments including untreated check replicated thrice in cotton during 2012 and 2013 at Tamil Nadu Agricultural University, Coimbatore. The experiments were conducted in a randomized block design with a plot size of $3 \times 7 \mathrm{~m}$ with three replications using the variety Surabhi. Applications of different treatments were imposed three times at an interval of 15 days during fruit formation stage using a high volume sprayer. The spraying was done during morning hours in such a way as to give uniform coverage on foliage and to avoid drift. A second spray was given 14 days after first application or considering the sucking pests and larval population crossing the ETL. The treatments were imposed commencing from $65^{\text {th }}$ day after planting with pneumatic knapsack sprayer using 500 litres of spray fluid per hectare. The data on percentage were transformed in to arcsine values and the population number into $\sqrt{ } \mathrm{X}+0.5$ before 
statistical analysis. The mean values were separated using Duncan's Multiple Range Test (DMRT). The treatments evaluated were as follows:

\begin{tabular}{lll}
\hline $\begin{array}{l}\text { S. } \\
\text { No }\end{array}$ & Name of the treatment & $\begin{array}{l}\text { Dose } \\
\mathrm{g} \mathrm{a.i} \mathrm{ha}^{-1}\end{array}$ \\
\hline 1 & Cyantraniliprole 10\% OD & 45 \\
2 & Cyantraniliprole 10\% OD & 60 \\
3 & Cyantraniliprole 10\% OD & 75 \\
4 & Cyantraniliprole 10\% OD & 90 \\
5 & Cyantraniliprole 10\% OD & 105 \\
6 & Chlorantraniliprole 20\% SC & 40 \\
7 & Acetamiprid 20 \% SP & 100 \\
8 & Untreated check & - \\
\hline
\end{tabular}

\section{Assessment of pest population}

\section{Sucking pests}

Observations on sucking insect pests viz., aphids, jassids, thrips, and whiteflies were made before as well as on 7 day after each application at three leaves per plant, one each from top, middle and bottom region from 5 randomly selected plants per plot, random, leaving border rows.

\section{Natural enemies (Spiders)}

In both the field trials, the natural enemies' fauna viz., spiders were recorded and the diversity of spiders was assessed at insecticide treated plots in cotton ecosystem from first and second experiment. The number of spiders was recorded on 5 randomly tagged plants per plot prior to application of insecticides and on 7 and 14 DAT in order to assess the effect of insecticides.

\section{Yield assessment}

Cotton yield per plot was recorded from each picking and pooled to arrive the total yield.
Finally, it was computed to quintal $\mathrm{ha}^{-1}$ and the same was subjected to statistical analysis.

\section{Results and discussion}

\section{Efficacy of cyantraniliprole $10 \%$ (w/v) OD against sucking pests}

\section{Jassids, Amrasca biguttula biguttula}

The first and second season experiment was conducted to evaluate the bioefficacy of cyantraniliprole $10 \%$ OD against jassids on cotton at TNAU, Coimbatore. After 7 days of first spraying, significant reduction in jassids population was observed in cyantraniliprole $10 \%$ OD treatments at 90 and $105 \mathrm{~g}$ a.i. ha ${ }^{-1}$ which recorded $8.90,8.57$ and $0.24,0.23$ jassids per three leaves plant ${ }^{-1}$, respectively. The standard insecticides viz. chlorantraniliprole $20 \% \mathrm{SC}$ at $40 \mathrm{~g}$ a.i. $\mathrm{ha}^{-1}$ and acetamprid $20 \% \mathrm{SP}$ at $100 \mathrm{~g}$ a.i. $\mathrm{ha}^{-1}$ recorded 61.23, 52.92 and $62.19,50.42$ per cent reduction of jassids populations in first and second seasons, respectively. After second application, cyantraniliprole 10\% OD at 90 and $105 \mathrm{~g}$ a.i. ha $^{-1}$ recorded 90.15, 90.15 and 95.87, 95.87 per cent reduction in jassids population at 7 days after spraying in first and second seasons, respectively (Table1).

\section{Whiteflies, Bemisia tabaci}

Seven days after application of cyantraniliprole $10 \%$ OD at 105 and $90 \mathrm{~g}$ a.i. $\mathrm{ha}^{-1}$ were on par with each other in first and second spray $(1.00,1.33$ and $0.30,0.47$ nos. three leaves ${ }^{-1}$ ) followed by $75 \mathrm{~g}$ a.i. $\mathrm{ha}^{-1}$ which recorded the lowest level of whitefly population (2.47 and 1.67 nos. five leaves ${ }^{-1}$ ), (Table 2). The same trend was noticed in second season also. More than 90 per cent reduction of whitefly was observed after 7 days of application of cyantraniliprole $10 \%$ OD at $105 \mathrm{~g}$ a.i. ha ${ }^{-1}$ and $90 \mathrm{~g}$ a.i. ha ${ }^{-1}$. 
Table.1 Effect of cyantraniliprole 10\% OD on leaf hopper, A.biguttula biguttula in cotton for season I and season II

\begin{tabular}{|c|c|c|c|c|c|c|c|c|c|c|c|}
\hline \multirow{4}{*}{$\begin{array}{l}\text { S. } \\
\text { No }\end{array}$} & \multirow{4}{*}{ Treatments } & \multirow{4}{*}{ PTC } & \multicolumn{9}{|c|}{ Leaf hopper population (Nos. 3 leaves $^{-1}$ ) (Mean of three replication) } \\
\hline & & & \multicolumn{4}{|c|}{ Season I } & \multirow{3}{*}{ PTC } & \multicolumn{4}{|c|}{ Season II } \\
\hline & & & \multicolumn{2}{|c|}{ I spray } & \multicolumn{2}{|c|}{ II spray } & & \multicolumn{2}{|c|}{ I spray } & \multicolumn{2}{|c|}{ II spray } \\
\hline & & & 7DAS & $\% \mathrm{RC}$ & 7DAS & $\% \mathrm{RC}$ & & 7DAS & $\% \mathrm{RC}$ & 7DAS & $\% \mathrm{RC}$ \\
\hline 1 & Cyantraniliprole $10 \%$ OD at $45 \mathrm{~g}$ a.i.ha ${ }^{-1}$ & 12.23 & $\begin{array}{c}11.77 \\
(3.41)^{\mathrm{c}}\end{array}$ & 59.85 & $\begin{array}{c}9.33 \\
(3.29)^{\mathrm{c}}\end{array}$ & 69.37 & 6.75 & $\begin{array}{c}2.69 \\
(1.78)^{b}\end{array}$ & 62.21 & $\begin{array}{c}1.70 \\
(1.48)^{b}\end{array}$ & 78.75 \\
\hline 2 & Cyantraniliprole $10 \%$ OD at $60 \mathrm{~g}$ a.i.ha ${ }^{-1}$ & 12.00 & $\begin{array}{l}10.77 \\
(3.32)^{\mathrm{c}}\end{array}$ & 59.85 & $\begin{array}{c}9.00 \\
(3.29)^{\mathrm{c}}\end{array}$ & 70.46 & 6.80 & $\begin{array}{c}2.50 \\
(1.73)^{b}\end{array}$ & 64.88 & $\begin{array}{c}1.30 \\
(1.34)^{b}\end{array}$ & 83.75 \\
\hline 3 & Cyantraniliprole $10 \%$ OD at $75 \mathrm{~g}$ a.i.ha ${ }^{-1}$ & 12.47 & $\begin{array}{c}10.00 \\
(3.32)^{c}\end{array}$ & 62.72 & $\begin{array}{c}8.00 \\
(2.91)^{\mathrm{b}}\end{array}$ & 73.74 & 6.87 & $\begin{array}{c}2.27 \\
(1.66)^{b}\end{array}$ & 68.11 & $\begin{array}{c}1.27 \\
(1.33)^{b}\end{array}$ & 84.12 \\
\hline 4 & Cyantraniliprole $10 \%$ OD at $90 \mathrm{~g}$ a.i.ha ${ }^{-1}$ & 12.00 & $\begin{array}{c}8.90 \\
(3.06)^{\mathrm{ab}}\end{array}$ & 66.82 & $\begin{array}{c}3.00 \\
(1.87)^{\mathrm{a}}\end{array}$ & 90.15 & 6.07 & $\begin{array}{c}0.24 \\
(0.86)^{\mathrm{a}}\end{array}$ & 96.62 & $\begin{array}{c}0.33 \\
(0.91)^{\mathrm{a}}\end{array}$ & 95.87 \\
\hline 5 & Cyantraniliprole $10 \%$ OD at $105 \mathrm{~g}$ a.i.ha ${ }^{-1}$ & 12.43 & $\begin{array}{c}8.57 \\
(2.99)^{\mathrm{a}}\end{array}$ & 68.05 & $\begin{array}{c}3.00 \\
(1.87)^{\mathrm{a}}\end{array}$ & 90.15 & 6.67 & $\begin{array}{c}0.23 \\
(0.85)^{\mathrm{a}}\end{array}$ & 96.76 & $\begin{array}{c}0.33 \\
(0.91)^{\mathrm{a}}\end{array}$ & 95.87 \\
\hline 6 & Chlorantraniliprole $20 \%$ SC at 40 g a.i.ha ${ }^{-1}$ & 12.67 & $\begin{array}{c}10.40 \\
(3.30)^{\mathrm{c}}\end{array}$ & 61.23 & $\begin{array}{c}9.13 \\
(3.10)^{b c}\end{array}$ & 70.03 & 6.47 & $\begin{array}{c}2.69 \\
(1.78)^{b}\end{array}$ & 62.19 & $\begin{array}{c}1.07 \\
(1.25)^{b}\end{array}$ & 86.62 \\
\hline 7 & Acetamiprid $20 \%$ SP at100 g a.i.ha ${ }^{-1}$ & 12.69 & $\begin{array}{c}12.63 \\
(3.59)^{d}\end{array}$ & 52.92 & $\begin{array}{l}10.20 \\
(3.27)^{\mathrm{c}}\end{array}$ & 66.52 & 6.27 & $\begin{array}{c}3.53 \\
(2.01)^{\mathrm{c}}\end{array}$ & 50.42 & $\begin{array}{c}2.27 \\
(1.66)^{\mathrm{c}}\end{array}$ & 71.62 \\
\hline 8 & Untreated check & 12.20 & $\begin{array}{c}26.83 \\
(5.31)^{\mathrm{e}}\end{array}$ & - & $\begin{array}{c}30.47 \\
(5.56)^{\mathrm{d}}\end{array}$ & - & 6.00 & $\begin{array}{c}7.12 \\
(2.76)^{d}\end{array}$ & - & $\begin{array}{c}8.00 \\
(2.91)^{\mathrm{d}}\end{array}$ & - \\
\hline
\end{tabular}

PTC- Pretreatment count

Values in parentheses are $\sqrt{x+0.5}$ transformed values

In a column means followed by a common letter are not significantly different by DMRT $(\mathrm{P}=0.05)$ 
Table.2 Effect of cyantraniliprole 10\% OD on whiteflies, B. tabaci in cotton for season I and season II

\begin{tabular}{|c|c|c|c|c|c|c|c|c|c|c|c|}
\hline \multirow{4}{*}{$\begin{array}{l}\text { S. } \\
\text { No }\end{array}$} & \multirow{4}{*}{ Treatments } & \multirow{4}{*}{ PTC } & \multicolumn{9}{|c|}{ Whiteflies (Nos. 3 leaves $^{-1}$ ) (Mean of three replication) } \\
\hline & & & \multicolumn{4}{|c|}{ Season I } & \multirow{3}{*}{ PTC } & \multicolumn{4}{|c|}{ Season II } \\
\hline & & & \multicolumn{2}{|c|}{ I spray } & \multicolumn{2}{|c|}{ II spray } & & \multicolumn{2}{|c|}{ I spray } & \multicolumn{2}{|c|}{ II spray } \\
\hline & & & 7DAS & $\% \mathrm{RC}$ & 7DAS & $\% \mathbf{R C}$ & & 7DAS & $\% \mathbf{R C}$ & 7DAS & $\% \mathbf{R C}$ \\
\hline 1 & Cyantraniliprole $10 \%$ OD at $45 \mathrm{~g}$ a.i.ha ${ }^{-1}$ & 4.67 & $\begin{array}{c}3.67 \\
(2.04)^{\mathrm{c}}\end{array}$ & 26.15 & $\begin{array}{c}2.67 \\
(1.78)^{\mathrm{bc}}\end{array}$ & 61.69 & 7.23 & $\begin{array}{c}5.27 \\
(2.40)^{\mathrm{c}}\end{array}$ & 38.50 & $\begin{array}{c}5.00 \\
(2.34)^{\mathrm{c}}\end{array}$ & 54.54 \\
\hline 2 & Cyantraniliprole $10 \%$ OD at $60 \mathrm{~g}$ a.i.ha ${ }^{-1}$ & 4.93 & $\begin{array}{c}2.57 \\
(1.75)^{b}\end{array}$ & 48.28 & $\begin{array}{c}2.00 \\
(1.58)^{b c}\end{array}$ & 71.30 & 7.47 & $\begin{array}{c}4.37 \\
(2.20)^{b c}\end{array}$ & 49.00 & $\begin{array}{c}4.00 \\
(2.12)^{b c}\end{array}$ & 63.63 \\
\hline 3 & Cyantraniliprole $10 \%$ OD at $75 \mathrm{~g}$ a.i.ha ${ }^{-1}$ & 4.67 & $\begin{array}{c}2.47 \\
(1.72)^{b}\end{array}$ & 50.30 & $\begin{array}{c}1.67 \\
(1.47)^{\mathrm{b}}\end{array}$ & 76.04 & 7.00 & $\begin{array}{c}3.27 \\
(1.94)^{b}\end{array}$ & 61.84 & $\begin{array}{c}3.00 \\
(1.87)^{b}\end{array}$ & 72.72 \\
\hline 4 & Cyantraniliprole $10 \%$ OD at $90 \mathrm{~g}$ a.i.ha ${ }^{-1}$ & 4.00 & $\begin{array}{c}1.33 \\
(1.35)^{\mathrm{a}}\end{array}$ & 73.23 & $\begin{array}{c}0.47 \\
(0.98)^{\mathrm{a}}\end{array}$ & 93.25 & 7.27 & $\begin{array}{c}1.77 \\
(1.50)^{\mathrm{a}}\end{array}$ & 79.34 & $\begin{array}{c}0.27 \\
(0.87)^{\mathrm{a}}\end{array}$ & 97.54 \\
\hline 5 & Cyantraniliprole $10 \%$ OD at $105 \mathrm{~g}$ a.i.ha ${ }^{-1}$ & 4.57 & $\begin{array}{c}1.00 \\
(1.22)^{\mathrm{a}}\end{array}$ & 79.89 & $\begin{array}{c}0.30 \\
(0.89)^{\mathrm{a}}\end{array}$ & 95.69 & 7.79 & $\begin{array}{c}1.03 \\
(1.23)^{\mathrm{a}}\end{array}$ & 87.98 & $\begin{array}{c}0.27 \\
(0.87) \mathrm{a}\end{array}$ & 97.54 \\
\hline 6 & Chlorantraniliprole $20 \%$ SC at 40 g a.i.ha ${ }^{-1}$ & 4.97 & $\begin{array}{c}2.70 \\
(1.78)^{b}\end{array}$ & 45.67 & $\begin{array}{c}2.33 \\
(1.68)^{\mathrm{bc}}\end{array}$ & 66.57 & 7.83 & $\begin{array}{c}3.33 \\
(1.95)^{b}\end{array}$ & 61.14 & $\begin{array}{c}4.67 \\
(2.27)^{\mathrm{c}}\end{array}$ & 57.54 \\
\hline 7 & Acetamiprid $20 \%$ SP at $100 \mathrm{~g}$ a.i.ha ${ }^{-1}$ & 4.03 & $\begin{array}{c}3.67 \\
(2.04)^{\mathrm{c}}\end{array}$ & 26.15 & $\begin{array}{c}3.00 \\
(1.87)^{\mathrm{d}}\end{array}$ & 56.95 & 7.49 & $\begin{array}{c}5.69 \\
(2.48)^{\mathrm{c}}\end{array}$ & 33.60 & $\begin{array}{c}5.27 \\
(2.40)^{\mathrm{c}}\end{array}$ & 52.09 \\
\hline 8 & Untreated check & 4.33 & $\begin{array}{c}4.97 \\
(2.33)^{\mathrm{d}}\end{array}$ & - & $\begin{array}{c}6.97 \\
(2.73)^{\mathrm{e}}\end{array}$ & - & 7.53 & $\begin{array}{c}8.57 \\
(3.81)^{\mathrm{d}}\end{array}$ & - & $\begin{array}{l}11.00 \\
(4.06)^{d}\end{array}$ & \\
\hline
\end{tabular}

PTC- Pretreatment count

Values in parentheses are $\sqrt{x+0.5}$ transformed values

In a column means followed by a common letter are not significantly different by DMRT $(\mathrm{P}=0.05)$ 
Table.3 Effect of cyantraniliprole 10\% OD on thrips, T. tabaci in cotton for season I and season II

\begin{tabular}{|c|c|c|c|c|c|c|c|c|c|c|c|}
\hline \multirow{4}{*}{$\begin{array}{l}\text { S. } \\
\text { No }\end{array}$} & \multirow{4}{*}{ Treatments } & \multirow{4}{*}{ PTC } & \multicolumn{9}{|c|}{ Thrips population (Nos. 3 leaves $^{-1}$ ) (Mean of three replication) } \\
\hline & & & \multicolumn{4}{|c|}{ Season I } & \multirow{3}{*}{ PTC } & \multicolumn{4}{|c|}{ Season II } \\
\hline & & & \multicolumn{2}{|c|}{ I spray } & \multicolumn{2}{|c|}{ II spray } & & \multicolumn{2}{|c|}{ I spray } & \multicolumn{2}{|c|}{ II spray } \\
\hline & & & 7DAS & $\% \mathbf{R C}$ & 7DAS & $\% \mathrm{RC}$ & & 7DAS & $\% \mathrm{RC}$ & 7DAS & $\% \mathbf{R C}$ \\
\hline 1 & Cyantraniliprole $10 \%$ OD at $45 \mathrm{~g}$ a.i.ha ${ }^{-1}$ & 5.00 & $\begin{array}{c}3.37 \\
(1.96)^{\mathrm{c}}\end{array}$ & 61.13 & $\begin{array}{c}2.30 \\
(1.14)^{b c}\end{array}$ & 80.78 & 5.20 & $\begin{array}{c}4.00 \\
(2.12)^{\mathrm{b}}\end{array}$ & 64.91 & $\begin{array}{c}3.69 \\
(2.04)^{\mathrm{bc}}\end{array}$ & 71.89 \\
\hline 2 & Cyantraniliprole $10 \%$ OD at $60 \mathrm{~g}$ a.i.ha ${ }^{-1}$ & 4.33 & $\begin{array}{c}2.00 \\
(1.58)^{\mathrm{b}}\end{array}$ & 76.93 & $\begin{array}{c}2.00 \\
(1.08)^{\mathrm{bc}}\end{array}$ & 83.29 & 5.57 & $\begin{array}{c}3.83 \\
(2.08)^{\mathrm{b}}\end{array}$ & 66.40 & $\begin{array}{c}3.00 \\
(1.87)^{b}\end{array}$ & 77.15 \\
\hline 3 & Cyantraniliprole $10 \%$ OD at $75 \mathrm{~g}$ a.i.ha ${ }^{-1}$ & 4.00 & $\begin{array}{c}1.87 \\
(1.53)^{\mathrm{b}}\end{array}$ & 78.43 & $\begin{array}{c}1.43 \\
(0.91)^{b}\end{array}$ & 88.05 & 5.23 & $\begin{array}{c}3.43 \\
(1.98)^{\mathrm{b}}\end{array}$ & 69.91 & $\begin{array}{c}2.67 \\
(1.78)^{b}\end{array}$ & 79.66 \\
\hline 4 & Cyantraniliprole $10 \%$ OD at $90 \mathrm{~g}$ a.i.ha ${ }^{-1}$ & 5.00 & $\begin{array}{c}0.57 \\
(0.87)^{\mathrm{a}}\end{array}$ & 93.42 & $\begin{array}{c}0.30 \\
(0.89)^{\mathrm{a}}\end{array}$ & 97.49 & 5.67 & $\begin{array}{c}1.07 \\
(1.25)^{\mathrm{a}}\end{array}$ & 90.61 & $\begin{array}{c}0.80 \\
(1.14)^{\mathrm{a}}\end{array}$ & 93.90 \\
\hline 5 & Cyantraniliprole $10 \%$ OD at $105 \mathrm{~g}$ a.i.ha ${ }^{-1}$ & 4.97 & $\begin{array}{c}0.27 \\
(0.87)^{\mathrm{a}}\end{array}$ & 96.88 & $\begin{array}{c}0.20 \\
(0.83)^{\mathrm{a}}\end{array}$ & 98.32 & 5.93 & $\begin{array}{c}0.93 \\
(1.19)^{\mathrm{a}}\end{array}$ & 91.84 & $\begin{array}{c}0.73 \\
(1.10)^{\mathrm{a}}\end{array}$ & 94.44 \\
\hline 6 & Chlorantraniliprole $20 \% \mathrm{SC}$ at $40 \mathrm{~g}$ a.i.ha ${ }^{-1}$ & 4.00 & $\begin{array}{c}2.00 \\
(1.58)^{b}\end{array}$ & 76.93 & $\begin{array}{c}2.90 \\
(1.18)^{\mathrm{bc}}\end{array}$ & 75.77 & 5.57 & $\begin{array}{c}4.50 \\
(2.23)^{\mathrm{bc}}\end{array}$ & 60.52 & $\begin{array}{c}4.00 \\
(2.12)^{\mathrm{c}}\end{array}$ & 69.53 \\
\hline 7 & Acetamiprid $20 \%$ SP at $100 \mathrm{~g}_{\text {a.i.ha }}{ }^{-1}$ & 4.33 & $\begin{array}{c}2.00 \\
(1.58)^{\mathrm{b}}\end{array}$ & 76.93 & $\begin{array}{c}2.00 \\
(1.22)^{\mathrm{bc}}\end{array}$ & 83.29 & 5.27 & $\begin{array}{c}3.67 \\
(2.04)^{b}\end{array}$ & 67.80 & $\begin{array}{c}3.27 \\
(1.94)^{\mathrm{bc}}\end{array}$ & 75.09 \\
\hline 8 & Untreated check & 4.67 & $\begin{array}{c}8.67 \\
(3.02)^{\mathrm{d}}\end{array}$ & - & $\begin{array}{c}11.97 \\
(3.53)^{\mathrm{d}}\end{array}$ & - & 5.50 & $\begin{array}{l}11.40 \\
(3.44)^{\mathrm{d}}\end{array}$ & - & $\begin{array}{c}13.13 \\
(3.70)^{\mathrm{d}}\end{array}$ & - \\
\hline
\end{tabular}

\section{PTC- Pretreatment count}

Values in parentheses are $\sqrt{x+0.5}$ transformed values

In a column means followed by a common letter are not significantly different by DMRT $(\mathrm{P}=0.05)$ 
Table.4 Effect of cyantraniliprole 10\% OD on aphids, A. gossypii in cotton for season I and season II

\begin{tabular}{|c|c|c|c|c|c|c|c|c|c|c|c|}
\hline \multirow{4}{*}{$\begin{array}{l}\text { S. } \\
\text { No }\end{array}$} & \multirow{4}{*}{ Treatments } & \multirow{4}{*}{ PTC } & \multicolumn{9}{|c|}{ Aphids population (Nos. 3 leaves $^{-1}$ ) (Mean of three replication) } \\
\hline & & & \multicolumn{4}{|c|}{ Season I } & \multirow{3}{*}{ PTC } & \multicolumn{4}{|c|}{ Season II } \\
\hline & & & \multicolumn{2}{|c|}{ I spray } & \multicolumn{2}{|c|}{ II spray } & & \multicolumn{2}{|c|}{ I spray } & \multicolumn{2}{|c|}{ II spray } \\
\hline & & & 7DAS & $\% \mathrm{RC}$ & 7DAS & $\% \mathbf{R C}$ & & 7DAS & $\% \mathrm{RC}$ & 7DAS & $\% \mathrm{RC}$ \\
\hline 1 & Cyantraniliprole $10 \%$ OD at $45 \mathrm{~g}$ a.i.ha ${ }^{-1}$ & 12.00 & $\begin{array}{c}9.27 \\
(3.12)^{\mathrm{bc}}\end{array}$ & 49.97 & $\begin{array}{c}7.00 \\
(2.73)^{\mathrm{b}}\end{array}$ & 66.23 & 10.00 & $\begin{array}{c}7.27 \\
(2.78)^{\mathrm{bc}}\end{array}$ & 56.01 & $\begin{array}{c}6.00 \\
(2.54)^{\mathrm{b}}\end{array}$ & 69.58 \\
\hline 2 & Cyantraniliprole $10 \%$ OD at $60 \mathrm{~g}$ a.i.ha ${ }^{-1}$ & 12.23 & $\begin{array}{c}9.00 \\
(3.08)^{b c}\end{array}$ & 51.43 & $\begin{array}{c}6.67 \\
(2.67)^{\mathrm{b}}\end{array}$ & 67.82 & 9.33 & $\begin{array}{c}7.00 \\
(2.73)^{b c}\end{array}$ & 57.65 & $\begin{array}{c}5.67 \\
(2.48)^{b}\end{array}$ & 71.26 \\
\hline 3 & Cyantraniliprole $10 \%$ OD at $75 \mathrm{~g}$ a.i.ha ${ }^{-1}$ & 12.29 & $\begin{array}{c}8.47 \\
(2.99)^{\mathrm{bc}}\end{array}$ & 54.29 & $\begin{array}{c}6.27 \\
(2.60)^{\mathrm{b}}\end{array}$ & 69.75 & 10.33 & $\begin{array}{c}6.47 \\
(2.64)^{\mathrm{bc}}\end{array}$ & 60.85 & $\begin{array}{c}5.27 \\
(2.40)^{\mathrm{b}}\end{array}$ & 73.28 \\
\hline 4 & Cyantraniliprole $10 \%$ OD at $90 \mathrm{~g}$ a.i.ha ${ }^{-1}$ & 12.47 & $\begin{array}{c}6.33 \\
(2.61)^{\mathrm{a}}\end{array}$ & 65.83 & $\begin{array}{c}4.53 \\
(2.24)^{\mathrm{a}}\end{array}$ & 78.14 & 9.67 & $\begin{array}{c}4.33 \\
(2.19)^{\mathrm{a}}\end{array}$ & 73.80 & $\begin{array}{c}3.53 \\
(2.00)^{\mathrm{a}}\end{array}$ & 82.10 \\
\hline 5 & Cyantraniliprole $10 \%$ OD at $105 \mathrm{~g}$ a.i.ha ${ }^{-1}$ & 12.73 & $\begin{array}{c}5.23 \\
(2.39)^{\mathrm{a}}\end{array}$ & 71.17 & $\begin{array}{c}4.00 \\
(2.12)^{\mathrm{a}}\end{array}$ & 80.70 & 9.87 & $\begin{array}{c}3.23 \\
(1.93)^{\mathrm{a}}\end{array}$ & 80.45 & $\begin{array}{c}3.00 \\
(1.87)^{\mathrm{a}}\end{array}$ & 84.79 \\
\hline 6 & Chlorantraniliprole $20 \% \mathrm{SC}$ at $40 \mathrm{~g}$ a.i.ha ${ }^{-1}$ & 12.69 & $\begin{array}{c}9.13 \\
(3.10)^{\mathrm{c}}\end{array}$ & 50.72 & $\begin{array}{c}7.47 \\
(2.82)^{b c}\end{array}$ & 63.96 & 10.67 & $\begin{array}{c}7.13 \\
(2.76)^{c}\end{array}$ & 56.86 & $\begin{array}{c}6.47 \\
(2.64)^{b c}\end{array}$ & 67.20 \\
\hline 7 & Acetamiprid $20 \%$ SP at100 g a.i.ha ${ }^{-1}$ & 12.00 & $\begin{array}{c}8.47 \\
(2.99)^{\mathrm{bc}}\end{array}$ & 54.29 & $\begin{array}{c}6.47 \\
(2.64)^{\mathrm{b}}\end{array}$ & 68.78 & 10.33 & $\begin{array}{c}6.47 \\
(2.64)^{\mathrm{bc}}\end{array}$ & 60.85 & $\begin{array}{c}5.47 \\
(2.44)^{b}\end{array}$ & 72.27 \\
\hline 8 & Untreated check & 12.47 & $\begin{array}{c}18.53 \\
(4.36)^{\mathrm{d}}\end{array}$ & - & $\begin{array}{c}20.73 \\
(4.60)^{\mathrm{d}}\end{array}$ & - & 10.00 & $\begin{array}{c}16.53 \\
(4.12)^{d}\end{array}$ & - & $\begin{array}{c}19.73 \\
(4.50)^{\mathrm{d}}\end{array}$ & - \\
\hline
\end{tabular}

PTC- Pretreatment count

Values in parentheses are $\sqrt{x+0.5}$ transformed values

In a column means followed by a common letter are not significantly different by DMRT $(\mathrm{P}=0.05)$ 
Table.5 Effect of cyantraniliprole 10\% OD on the yield of cotton

\begin{tabular}{|c|c|c|c|}
\hline \multirow[t]{2}{*}{ Treatments } & \multirow{2}{*}{$\begin{array}{c}\text { Dose } \\
\left(\mathrm{g} \mathrm{a.i.ha}^{-1}\right)\end{array}$} & \multicolumn{2}{|c|}{$\begin{array}{l}\text { Kapas Yield } \\
\quad\left(\mathbf{q} \mathbf{h a}^{-1}\right)\end{array}$} \\
\hline & & Season I & Season II \\
\hline Cyantraniliprole 10\% OD & 45 & $15.32^{\mathrm{c}}$ & $14.32^{\mathrm{c}}$ \\
\hline Cyantraniliprole $10 \%$ OD & 60 & $16.80^{\mathrm{b}}$ & $16.87^{\mathrm{b}}$ \\
\hline Cyantraniliprole $10 \%$ OD & 75 & $16.20^{\mathrm{b}}$ & $16.20^{\mathrm{b}}$ \\
\hline Cyantraniliprole $10 \%$ OD & 90 & $19.45^{\mathrm{a}}$ & $18.45^{\mathrm{a}}$ \\
\hline Cyantraniliprole $10 \%$ OD & 105 & $19.88^{\mathrm{a}}$ & $18.47^{\mathrm{a}}$ \\
\hline Chlorantraniliprole $20 \%$ SC & 40 & $15.47^{\mathrm{c}}$ & $14.49^{\mathrm{c}}$ \\
\hline Acetamiprid $20 \% \mathrm{SP}$ & 100 & $12.23^{\mathrm{d}}$ & $11.43^{\mathrm{d}}$ \\
\hline Untreated check & - & $12.08^{\mathrm{d}}$ & $10.02^{\mathrm{e}}$ \\
\hline
\end{tabular}

*Mean of three plots; in a column,

Means followed by a common letter(s) are not significantly different by DMRT $(\mathrm{P}=0.05)$

Table.6 Phytotoxicity of cyantraniliprole 10 OD on cotton

\begin{tabular}{|c|c|c|c|c|c|c|}
\hline \multirow[b]{2}{*}{ Treatment } & \multirow[b]{2}{*}{$\begin{array}{c}\text { Dose } \\
\left(\mathrm{g} \mathrm{a.i.ha}^{-1}\right)\end{array}$} & \multicolumn{5}{|c|}{ Phytotoxic symptoms* } \\
\hline & & $\begin{array}{l}\text { Injury to } \\
\text { leaf tip }\end{array}$ & Wilting & $\begin{array}{l}\text { Vein } \\
\text { clearing }\end{array}$ & Necrosis & $\begin{array}{c}\text { Epinasty and } \\
\text { Hyponasty }\end{array}$ \\
\hline Cyantraniliprole $10 \%$ OD & 90 & 0 & 0 & 0 & 0 & 0 \\
\hline Cyantraniliprole $10 \%$ OD & 180 & 0 & 0 & 0 & 0 & 0 \\
\hline Cyantraniliprole $10 \%$ OD & 360 & 0 & 0 & 0 & 0 & 0 \\
\hline Untreated check & - & 0 & 0 & 0 & 0 & 0 \\
\hline
\end{tabular}

*Symptoms for phytotoxicity observed on 1, 3, 7, 10, 14 days after spray

0 - No phytotoxicity 
Table.7 Effect of cyantraniliprole 10OD on spiders in cotton for season I and season II

\begin{tabular}{|c|c|c|c|c|c|c|c|c|c|c|c|}
\hline \multirow{4}{*}{$\begin{array}{l}\text { S. } \\
\text { No }\end{array}$} & \multirow{4}{*}{ Treatments } & \multirow{4}{*}{ PTC } & \multicolumn{9}{|c|}{ Spiders population (No./ 5 plants)* (Mean of three replication) } \\
\hline & & & \multicolumn{4}{|c|}{ Season I } & \multirow{3}{*}{ PTC } & \multicolumn{4}{|c|}{ Season II } \\
\hline & & & \multicolumn{2}{|c|}{ I spray } & \multicolumn{2}{|c|}{ II spray } & & \multicolumn{2}{|c|}{ I spray } & \multicolumn{2}{|c|}{ II spray } \\
\hline & & & 7DAS & 14DAS & 7DAS & 14DAS & & 7DAS & 14DAS & 7DAS & 14DAS \\
\hline 1 & Cyantraniliprole $10 \%$ OD at $45 \mathrm{~g}$ a.i.ha ${ }^{-1}$ & 3.67 & $\begin{array}{c}4.87 \\
(2.32)^{\mathrm{a}}\end{array}$ & $\begin{array}{c}6.00 \\
(2.55)^{\mathrm{ab}}\end{array}$ & $\begin{array}{c}6.33 \\
(2.61)^{\mathrm{ab}}\end{array}$ & $\begin{array}{c}7.87 \\
(2.89)^{\mathrm{ab}}\end{array}$ & 4.33 & $\begin{array}{c}4.33 \\
(2.20)^{\mathrm{ab}}\end{array}$ & $\begin{array}{c}5.67 \\
(2.48)^{\mathrm{ab}}\end{array}$ & $\begin{array}{c}6.33 \\
(2.61)^{b}\end{array}$ & $\begin{array}{c}7.77 \\
(2.88)^{\mathrm{ab}}\end{array}$ \\
\hline 2 & Cyantraniliprole $10 \%$ OD at $60 \mathrm{~g}$ a.i.ha ${ }^{-1}$ & 3.80 & $\begin{array}{c}4.67 \\
(2.27)^{\mathrm{ab}}\end{array}$ & $\begin{array}{c}5.60 \\
(2.47)^{\mathrm{ab}}\end{array}$ & $\begin{array}{c}6.00 \\
(2.55)^{\mathrm{bc}}\end{array}$ & $\begin{array}{c}7.00 \\
(2.74)^{\mathrm{bcd}}\end{array}$ & 4.00 & $\begin{array}{c}4.00 \\
(2.12)^{\mathrm{abc}}\end{array}$ & $\begin{array}{c}5.50 \\
(2.45)^{\mathrm{ab}}\end{array}$ & $\begin{array}{c}6.07 \\
(2.56)^{\mathrm{bc}}\end{array}$ & $\begin{array}{c}7.33 \\
(2.80)^{\mathrm{abc}}\end{array}$ \\
\hline 3 & Cyantraniliprole $10 \%$ OD at $75 \mathrm{~g}$ a.i.ha ${ }^{-1}$ & 3.67 & $\begin{array}{c}4.33 \\
(2.20)^{\mathrm{ab}}\end{array}$ & $\begin{array}{c}5.33 \\
(2.41)^{\mathrm{b}}\end{array}$ & $\begin{array}{c}6.00 \\
(2.55)^{\mathrm{bc}}\end{array}$ & $\begin{array}{c}7.33 \\
(2.80)^{\mathrm{abc}}\end{array}$ & 3.33 & $\begin{array}{c}4.00 \\
(2.12)^{\mathrm{abc}}\end{array}$ & $\begin{array}{c}5.50 \\
(2.45)^{\mathrm{ab}}\end{array}$ & $\begin{array}{c}6.10 \\
(2.57)^{\mathrm{bc}}\end{array}$ & $\begin{array}{c}7.30 \\
(2.79)^{\mathrm{abc}}\end{array}$ \\
\hline 4 & Cyantraniliprole $10 \%$ OD at $90 \mathrm{~g}$ a.i.ha ${ }^{-1}$ & 2.87 & $\begin{array}{c}4.33 \\
(2.20)^{\mathrm{ab}}\end{array}$ & $\begin{array}{c}5.00 \\
(2.35)^{\mathrm{b}}\end{array}$ & $\begin{array}{c}5.87 \\
(2.52)^{\mathrm{bc}}\end{array}$ & $\begin{array}{c}6.60 \\
(2.66)^{\text {cde }}\end{array}$ & 3.67 & $\begin{array}{c}3.50 \\
(2.00)^{\mathrm{bc}}\end{array}$ & $\begin{array}{c}5.37 \\
(2.42)^{\mathrm{bc}}\end{array}$ & $\begin{array}{c}5.87 \\
(2.52)^{\mathrm{bc}}\end{array}$ & $\begin{array}{c}6.67 \\
(2.68)^{\mathrm{cd}}\end{array}$ \\
\hline 5 & Cyantraniliprole $10 \%$ OD at $105 \mathrm{~g}$ a.i.ha ${ }^{-1}$ & 3.17 & $\begin{array}{c}4.00 \\
(2.12)^{\mathrm{bc}}\end{array}$ & $\begin{array}{c}4.67 \\
(2.27)^{\mathrm{bc}}\end{array}$ & $\begin{array}{c}5.33 \\
(2.41)^{\mathrm{c}}\end{array}$ & $\begin{array}{c}6.00 \\
(2.55)^{\mathrm{e}}\end{array}$ & 3.67 & $\begin{array}{c}3.17 \\
(1.92)^{\mathrm{c}}\end{array}$ & $\begin{array}{c}5.30 \\
(2.41)^{\mathrm{bc}}\end{array}$ & $\begin{array}{c}5.67 \\
(2.48)^{\mathrm{bc}}\end{array}$ & $\begin{array}{c}6.33 \\
(2.61)^{\mathrm{d}}\end{array}$ \\
\hline 6 & Chlorantraniliprole $20 \% \mathrm{SC}$ at $40 \mathrm{~g}$ a.i.ha ${ }^{-1}$ & 2.93 & $\begin{array}{c}4.00 \\
(2.12)^{\mathrm{bc}}\end{array}$ & $\begin{array}{c}5.00 \\
(2.35)^{\mathrm{bc}}\end{array}$ & $\begin{array}{c}5.67 \\
(2.48)^{\mathrm{bc}}\end{array}$ & $\begin{array}{c}7.00 \\
(2.74)^{b c d}\end{array}$ & 4.33 & $\begin{array}{c}3.67 \\
(2.04)^{\mathrm{bc}}\end{array}$ & $\begin{array}{c}5.00 \\
(2.35)^{\mathrm{bc}}\end{array}$ & $\begin{array}{c}5.97 \\
(2.54)^{\mathrm{bc}}\end{array}$ & $\begin{array}{c}7.00 \\
(2.74)^{\mathrm{bcd}}\end{array}$ \\
\hline 7 & Acetamiprid $20 \%$ SP at100 g a.i.ha ${ }^{-1}$ & 3.00 & $\begin{array}{c}3.33 \\
(1.96)^{\mathrm{c}}\end{array}$ & $\begin{array}{c}5.00 \\
(2.35)^{\mathrm{bc}}\end{array}$ & $\begin{array}{c}5.33 \\
(2.41)^{\mathrm{c}}\end{array}$ & $\begin{array}{c}6.33 \\
(2.61)^{\mathrm{de}}\end{array}$ & 3.33 & $\begin{array}{c}3.33 \\
(1.96)^{\mathrm{c}}\end{array}$ & $\begin{array}{c}4.67 \\
(2.27)^{\mathrm{c}}\end{array}$ & $\begin{array}{c}5.33 \\
(2.41)^{\mathrm{c}}\end{array}$ & $\begin{array}{c}6.33 \\
(2.61)^{\mathrm{d}}\end{array}$ \\
\hline 8 & Untreated check & 3.00 & $\begin{array}{c}5.00 \\
(2.35)^{\mathrm{a}}\end{array}$ & $\begin{array}{c}6.33 \\
(2.61)^{\mathrm{a}}\end{array}$ & $\begin{array}{c}7.00 \\
(2.74)^{\mathrm{a}}\end{array}$ & $\begin{array}{c}8.00 \\
(2.92)^{\mathrm{a}}\end{array}$ & 3.67 & $\begin{array}{c}4.80 \\
(2.30)^{\mathrm{a}}\end{array}$ & $\begin{array}{c}6.37 \\
(2.62)^{\mathrm{a}}\end{array}$ & $\begin{array}{c}7.33 \\
(2.80)^{\mathrm{a}}\end{array}$ & $\begin{array}{c}8.00 \\
(2.92)^{\mathrm{a}}\end{array}$ \\
\hline
\end{tabular}

PTC- Pretreatment count

Values in parentheses are $\sqrt{x+0.5}$ transformed values

In a column means followed by a common letter are not significantly different by DMRT $(\mathrm{P}=0.05)$ 


\section{Thrips, Thrips tabaci}

Significant reduction in thrips population was observed in cyantraniliprole $10 \%$ OD treatments at 90 and $105 \mathrm{~g}$ a.i. $\mathrm{ha}^{-1}$ which recorded $0.57,0.30$ and $0.27,0.20$ thrips three leaves plants ${ }^{-1}$ after first and second spray, respectively at 7 DAS.

While the untreated check recorded 8.67 and 11.97 thrips three leaves ${ }^{-1}$ plant $^{-1}$ at 7 days after first and second application respectively.

During second season, cyantraniliprole $10 \%$ OD at 90 and $105 \mathrm{~g}$ a.i. $\mathrm{ha}^{-1}$ recorded 90.61 , 91.84 and $93.90,94.44$ per cent reduction in thrips population after first and second spray at 7 DAS, respectively (Table 3 ).

\section{Aphid, Aphis gossypii}

The pretreatment population of aphids varied from 12.0 to 12.73 nos. five leaves ${ }^{-1}$ (Table 4). After 7 days of first spraying, significant reduction in aphids population was observed in cyantraniliprole $10 \%$ OD treatments at 105 and $90 \mathrm{~g}$ a.i. $\mathrm{ha}^{-1}$ which recorded $71.17,65.83$ and $80.70,78.14$ per cent reduction of aphids population in first season at first and second spray, respectively. Whereas, the standard insecticides viz., chlorantraniliprole $20 \%$ SC at $40 \mathrm{~g}$ a.i. $\mathrm{ha}^{-1}$ and acetamprid $20 \% \mathrm{SP}$ at 100 g a.i. $\mathrm{ha}^{-1}$ recorded 50.72, 54.29 and 63.96, 68.78 per cent reduction in aphids population in first season at first and second spray, respectively.

While second season, cyantraniliprole $10 \%$ $\mathrm{OD}$ at 90 and $105 \mathrm{~g}$ a.i. $\mathrm{ha}^{-1}$ recorded 73.80 , 80.45 and $82.10,84.79$ per cent reduction in aphids population at 7 days after spraying, respectively whereas chlorantraniliprole $20 \%$ SC and acetamprid 20\% SP recorded 56.86, 60.85 and $67.20,72.27$ per cent reduction in aphids population at 7 days after first and second spraying, respectively.

\section{Phytotoxicity of cyantraniliprole 10\% (w/v) OD in cotton}

Results of the field trial conducted at TNAU of Coimbatore district revealed that cotton plants sprayed with cyantraniliprole $10 \mathrm{OD}$ at 90, 180 and $360 \mathrm{~g}$ a.i. $\mathrm{ha}^{-1}$ doses did not show any phytotoxic symptoms like leaf tip injury, vein clearing, wilting, necrosis, epinasty and hyponasty. The phytotoxic rating of zero in all the doses was observed on 1, 3, 7, 10 and 14 DAS (Table 5).

Bio-safety of cyantraniliprole $10 \%(w / v)$ OD on coccinellids and spiders

\section{Spiders}

In the first and second field experiment, pretreatment population of spiders ranged from 2.87 to 3.80 and 3.33 to 4.33 per five plants, respectively. The lowest concentration of cyantraniliprole $10 \mathrm{OD}$ at $45 \mathrm{~g}$ a.i. $\mathrm{ha}^{-1}$ harbored maximum spider population of 4.87, 6.00 and $6.33,7.87$ at 7 and $14^{\text {th }}$ days after first and second spray per five plants followed by cyantraniliprole $10 \mathrm{OD}$ at $60 \mathrm{~g}$ a.i. $\mathrm{ha}^{-1}$ (4.67, 5.60 and 6.00, 7.00 nos. after first and second spray, respectively) and cyantraniliprole $10 \mathrm{OD}$ at $75 \mathrm{~g}$ a.i. $\mathrm{ha}^{-1}$ (4.33, 6.00 and 5.33, 7.33 nos. after first and second spray, respectively). After second season, cyantraniliprole 10 OD treated plots at 75, 60 and $45 \mathrm{~g}$ a.i. $\mathrm{ha}^{-1}$ recorded the maximum spider population per five plants which was on par with untreated check (Table 6).

The results of the field experiments on cotton revealed that after second application cyantraniliprole $10 \%$ OD at 90 and $105 \mathrm{~g}$ a.i. $\mathrm{ha}^{-1}$ recorded 90.15, 90.15 and 95.87, 95.87 per cent reduction in jassids population at 7 days after spraying in first and second seasons, respectively. Cyantraniliprole 10\% $\mathrm{OD}$ at 105 and $90 \mathrm{~g}$ a.i. ha ${ }^{-1}$ were on par with each other in first and second spray (1.00, 
1.33 and $0.30,0.47$ nos. three leaves ${ }^{-1}$ ) and Ninety per cent reduction of whitefly population was observed after 7 days of. This is in conformity with finding of Patel et al., 2014 stated that after $3^{\text {rd }}$ day, newer molecule of cyantraniliprole at $105 \mathrm{~g}$ a.i. ha ${ }^{-1}$ was found significantly more effective than all the doses of cyantraniliprole, except $90 \mathrm{~g}$ a.i. ha ${ }^{-1}$ and recorded lower population of whitefly i.e. 0.92 and 0.75 per leaf after $3^{\text {rd }}$ dayand 0.26 and 0.14 per leaf after $7^{\text {th }}$ day of spray during 2010- 11 and 2011-12, respectively.

In case of cyantraniliprole at $90 \mathrm{~g}$ a.i. $\mathrm{ha}^{-1}$, it was 1.01 and 0.82 after $3^{\text {rd }}$ day of spray and 0.31 and 0.17 per leaf after $7^{\text {th }}$ day of spray. Similarly, cyantraniliprole (90 and $105 \mathrm{~g}$ a.i. $\mathrm{ha}^{-1}$ ) was also effective against whitefly on okra, brinjal and tomato as reported by Patel et al., 2011, Patel and Kher, 2012.

After seven days of first spraying, significant reduction in aphids population was observed in cyantraniliprole $10 \%$ OD treatments at 90 and $105 \mathrm{~g}$ a.i. ha ${ }^{-1}$ which recorded 71.17 , 65.83 and $80.70,78.14$ per cent reduction of aphids population in first season at first and second spray, respectively. The superiority of cyantraniliprole against $A$. gossypii revealed in present study is in accordance with reports of Patel et al., 2014 who reported that among the insecticidal treatments, cyantraniliprole at highest dose i.e. $105 \mathrm{~g}$ a.i. $\mathrm{ha}^{-1}$ significantly reduced the aphid population and recorded 1.38 and 2.22 per leaf at $3^{\text {rd }}$ day after spray and 0.17 and 0.42 per leaf at $7^{\text {th }}$ day after spray during 2010-11 and2011-12, respectively.

The treatment of cyantraniliprole at $90 \mathrm{~g}$ a.i.ha ${ }^{-1}$ stood next to this and was at par with the highest dose cyantraniliprole of $105 \mathrm{~g}$ a.i.ha ${ }^{-1}$. Misra (2013) reported that cyantraniliprole (HGW86) 10 per cent OD at the rate of 90 and $105 \mathrm{~g}$ a.i. ha $^{-1}$ (4.87 and 7.40) with $98.79,93.85$ and 95.95 per cent reduction in aphid population over untreated control, respectively. Mandal (2012) who reported that cyantraniliprole at 90 and $105 \mathrm{~g}$ a.i.ha ${ }^{-1}$ was more effective in reducing the pest population in tomato.

The anthranillic diamide insecticide group possesses anti-feedant properties that differ between chemicals of this group and insects (Gonzales-Coloma et al., 1999) which might be the reason of record of low population of aphids. Cyantraniliprole 10\% OD at 90 and $105 \mathrm{~g}$ a.i. $\mathrm{ha}^{-1}$ recorded 90.61, 91.84 and 93.90, 94.44 per cent reduction in thrips population after first and second spray after second season at 7 DAS, respectively.

Patel (2014) revealed that After the $3^{\text {rd }}$ and $7^{\text {th }}$ day of spray, cyantraniliprole at105 $\mathrm{g}$ a.i./ha found significantly more effective in controlling thrips and it was at par with cyantraniliprole at $90 \mathrm{~g}$ a.i./ha. According to Misra (2012), both the doses of cyantraniliprole i.e. 105 and $90 \mathrm{~g}$ a.i./ha were found equally effective against $T$. tabaci infesting tomato. This is in agreement with the present finding.

The lowest concentration of cyantraniliprole $10 \mathrm{OD}$ at $45 \mathrm{~g}$ a.i. $\mathrm{ha}^{-1}$ harbored maximum spider population of $4.87,6.00$ and $6.33,7.87$ at 7 and $14^{\text {th }}$ days after first and second spray per five plants followed by cyantraniliprole $10 \mathrm{OD}$ at $75 \mathrm{~g}$ a.i. ha ${ }^{-1}$. Cyantraniliprole 10 OD treated plots at $90,75,60$ and $45 \mathrm{~g}$ a.i. ha ${ }^{1}$ recorded the maximum spider population per five plants which was onpar with untreated check. Anon (2013) was revealed that Cyazypyr played a role to maximize the population of natural enemies by conserving predators and parasitoids and there thereby giving excellent control of whiteflies, aphids, thrips, leafminers, beetles and Lepidoptera on various vegetables and fruit crops. Flubendiamide $480 \mathrm{SC}$ was reported as safe to the natural enemies like spiders and lady bird beetles (Latif et al., 2009) (Table 7). 


\section{Acknowledgements}

The authors are grateful to E.I Dupont for providing financial support and the chemicals used for this study.

\section{References}

Anonymous. 2012. DuPont cyazypyr ${ }^{\mathrm{TM}}$ insect control. Tech. Bull., E.I., Du Pont de Nemours and Company. p. 4.

Anonymous. 1997. Pesticides Production Current Status. Pesticide Information, 22(4): 36-38.

Armes, N.J., Banerjee, S.K., De Souza, K.R., Jadhav, D.R., King, A.B.S., Kranthi, K.R., Regupathy, A., Surilivelu, T. and Venugopal Rao, N. 1994. Insecticticide resistance in Helicoverpa armigera in India: Recent Developments. Brighton Crop Protection Conference - Pests and Dis., 437 - 442.

Awasthi, M.D., Sharma, D. and Ahuja, A.K. 2002. Monitoring of horticultural ecosystem: Orchard soil and water bodies for pesticide residues around North Bangalore. Pestic. Res. J., 14(2): 286-291.

Gonzales-Coloma, A., Gutierrez, C., Hubner, H., Achenbach, H., Terrero, D. and Fraga, B.M. 1999. Selective insect antifeedant and toxic action of ryanoid diterpenes. J. Agric. Food Chem., 47: 4419-4424.

Hardin, M.R., Benrey, B.M., Colt, L., William, K.R., George and Barbosa, P. 1995. Arthropod pest resurgence: an overview of potential mechanisms. Crop Protection, 14(1): 3-18.

Latif, M.A., Rahman, M.M. and Hossain, M.M. 2009. "Effect of Flubendiamide and Some Other Insecticides on Arthropod's BiodiversityUsed to Control Brinjal Shoot and Fruit Borer (Leucinodes orbonalis G.)", Intl. J. Agric. Envir. Biotech., 2: 173-179.
Mandal, S.K. 2012. Bio-efficacy of cyazypyr $10 \%$ OD, a new anthranilicdiamide insecticide against the insect pests of tomato and its impact on natural enemies and crop health. Acta Phytopathologica et Entomologica Hungarica, 47(2): 233-249.

McCaffery, A.R., King, A.B.S., Walker, A.J. and Elnayir, H. 1989. Resistance to synthetic pyrethroids in the bollworm Heliothis armigera from Andhra Pradesh, India. Pestic. Sci., 27: 65-76.

Misra, H.P. 2012. Bio-efficacy of chlorantraniliprole against shoot and fruit borer of brinjal, Leucinodesorbonalis Guenee. J. Insect Sci., 24(1): 60-64.

Patel, J.J., Patel, H.C. and Kathiria, K.B. 2011. Testing the bioefficacy and phytotoxicity of cyantraniliprole 10\% OD against pestsof tomato. Final report, Main Vegetable Research Station, Anand Agricultural University, Anand, pp. 16-18.

Patel, R.D., Bharpoda, T.M. and Borad, P.K. 2012. Larvicidal efficacy of cyantraniliprole against Spodopteralitura (Fabricius) in cotton. An Int. e-J., 1(4): 530-533.

Patel, R.D., Bharpoda, T.M., Patel, N.B. and Borad, P.K. 2014. Bio-efficacy of cyantraniliprole $\quad 10 \%$ OD- an anthranilicdiamide insecticide against sucking pests of cotton.The Bioscan 9(1): 89-92.

Rolando, T., Francesco, M. and Massimo, B. 1982. Simultaneous determination of organochlorine, organophosphorus, pthalamide, oxazolidine, pyrethroid, $\mathrm{N}$ methyl carbamate, benzimidazole and thioureidobe benzene pesticide in agricultural products. Bull Chem Unione Ital. Lab. Prove Parte Sci., 33: $69-112$.

Taley, Y.M., Thote, R.L. and Nimbalkar, S.A. 1988. Assessment of losses due to 
insect pests of cotton and benefit of protection schedule. PKV. Res. J., 12: 88-91.

Venugopal, M.S. 1988. Non-pesticidal ecofriendly management of pests of cotton in Tamil Nadu. In: Workshop Proc. Non-pesticidal management of cotton and pigeon pea pests, April 10-11, (Chari, MS, Qayum MA, Sanghi NK and Sastri MV (eds.), Center for World Solidarity, Secunderabad and National Institute of Agrl. Extension Management, Hyderabad.

\section{How to cite this article:}

Karthik, P., T. Thiruveni, K. Indirakumar, K. Gunasekaran, S. Kuttalam and Srinivasan, V.M. 2017. Bioefficacy and Safety of Cyantraniliprole $10 \%$ (W/V) OD against Sucking Pests in Cotton. Int.J.Curr.Microbiol.App.Sci. 6(2): 1405-1417.

doi: http://dx.doi.org/10.20546/ijcmas.2017.602.159 